\title{
The Twinned Crystal Structure of Zinc(II) Acetylacetonate Trimer
}

\author{
Alexander Hübner · Daniel Strobusch • \\ Hans-Wolfram Lerner · Michael Bolte
}

Published online: 13 September 2008

(C) Springer Science+Business Media, LLC 2008

Erratum to: J Chem Crystallogr

DOI 10.1007/s10870-008-9422-y

Daniel Strobusch's name was inadvertently misspelled in the original article.

The online version of the original article can be found under doi:10.1007/s10870-008-9422-y.

\footnotetext{
A. Hübner · D. Strobusch · H.-W. Lerner · M. Bolte $(\square)$ Institut für Anorganische Chemie, J. W. Goethe-Universität Frankfurt, Max-von-Laue-Str. 7, 60438 Frankfurt/Main, Germany

e-mail: bolte@chemie.uni-frankfurt.de
} 